\title{
Micro-usinage par impulsions laser UV : diagnostic d'interaction
}

\author{
C. Stauter, P. Gérard, J. Fontaine et T. Engel
}

\author{
Laboratoire d'Application des Lasers, ENSAIS, 24 boulevard de la Victoire, 67084 \\ Strasbourg cedex, France et \\ IREPA-LASER, Pôle API, 67400 Illkirch, France
}

\begin{abstract}
Résumé:
Lors de l'ablation de céramiques et de polymères à l'aide de lasers à impulsions courtes dans le domaine UV, des ondes de chocs sont générées dans l'air. A basse fluence, nous observons que le taux d'ablation est fonction linéaire de l'intensité acoustique. Celle-ci est mesurée à l'aide d'une technique de déllexion de faisceau ou par un microphone. Un contrôle de procédé par voie acoustique est envisagé.
\end{abstract}

\section{Introduction}

Le procédé d'ablation par laser impulsionnel est très prometteur pour les applications de traitement de surfaces et de micro-usinage car il concerne une très large gamme de matériaux: métaux, céramiques, polymères. Cependant, pour les applications de micro-usinage, du fait des fluctuations de puissance des impulsions laser et des inhomogénéités de surface des matériaux, il est nécessaire de développer un système permettant de contrôler de façon précise la quantité de matière ablatée. Dans cette étude, nous évaluons l'intérêt d'un contrôle de procédé par voie acoustique en établissant un lien entre le taux d'ablation et l'intensité des signaux acoustiques générés lors des tirs laser.

\section{Procédure expérimentale}

Le dispositif de mesures est présenté figure 1. Dans cette étude, nous avons utilisé un liaser $\mathrm{Nd}$ : YAG triplé $(355 \mathrm{~nm})$, et un laser à excimère $\mathrm{KrF}(248 \mathrm{~nm})$. Un joulemètre mesure l'énergie du faisceáu par prélèvenent, après quoi un diaphragme (D) permet de sélectionner une portion du faisceau d'intensité uniforme, qu'une lentille (L1) focalise sur un spot d'environ $0.2 \mathrm{~mm}$ de diamètre sur l'échantillon (E). Un faisceau laser $\mathrm{He}-\mathrm{Ne}$, passant à quelques millimètres parallèlement à la surface de l'échantillon est utilisé pour détecter par la technique de déflexion photo-' acoustique l'onde de choc créée par l'inpact de

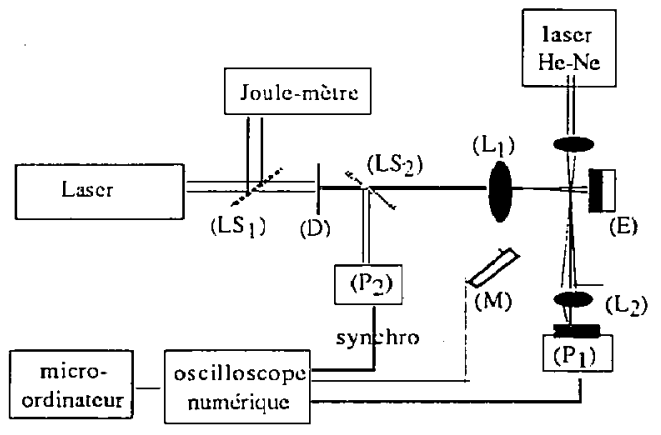
l'impulsion laser. La déflexion du faisceau-sonde est détectée à l'aide d'une photodiode (P1) placée derrière un ensemble lentille et lame de couteilu (L2). Une autre photodiode (P2) recevant une fraction du faisceau de puissance est utilisée pour le déclenchement de l'oscilloscope numérique. Un microphone (M) permet de relever d'une autre manière l'intensité acoustique des ondes de choc [1,2].

\section{Résultats}

Nous étudions l'ablation d'une céramique $\mathrm{Si}_{3} \mathrm{~N}_{4}$ par des impulsions d'un laser à excimère $\mathrm{Kr} F$. Les impulsions laser ont une durée de 22 ns. Nous réalisons un nombre donné de tirs sur l'échantillon et mesurons à l'aide d'un microscope la profondeur des trous ainsi créés. Nous traçons alors la courbe d'ablation en fonction de l'irradiance. Celle-ci est présentée sur la figure 2. A chaque tir laser, la température de la surface de l'échantillon augmente très rapidement. L'air au-dessus de la surface se dilate, ce qui génère alors une onde de choc. Nous avons cherché à déterminer la quantité d'énergie du faisceau laser transférée dans cette onde de choc. Pour cela, nous avons mesuré le temps de vol de 
cette onde de choc. Dans notre dispositif expérimental le temps de vol est la durée qui s'écoule entre l'instant du tir laser et le moment où nous observons la déflexion photo-acoustique du faisceau sonde. Connaissant la distance séparant le faisceau sonde de la surface de l'échantillon, nous calculons la vitesse de propagation de l'onde de choc. Ces mesures sont réalisées sur oscilloscope numérique. La distance d'observation de cette onde de choc est telle que nous nous trouvons dans la région de transition acoustique dans laquelle l'onde de choc peut se décrire par le modèle de Jones [3]. Ce modèle nous permet à partir de la mesure du temps de vol de remonter à l'énergie qui a créé l'onde de choc. Ceci nous permet d'obtenir le graphe de la figure 3 donnant l'évolution de l'énergie d'onde de choc en fonction de l'irradiance. Les réglages précis de la technique de déflexion photo-acoustique ainsi que les traitements des signaux associés rendent celle-ci difficilement utilisable sur site industriel. Nous avons donc cherché à mesurer l'énergie des ondes de chocs à l'aide d'un simple microphone. Ceci est possible en étalonnant le microphone pour une distance donnée d d'observation par rapport à la cible. Une courbe d'étalonnage est présentée figure 4, pour différentes distances d. A partir des données des figures 2,3 et 4, nous pouvons tracer la courbe représentant le taux d'ablation en fonction de l'amplitude du signal du microphone (figure 5). Il est ainsi possible d'utiliser l'amplitude des signaux du microphone pour contrôler en temps réel le procédé d'ablation laser.

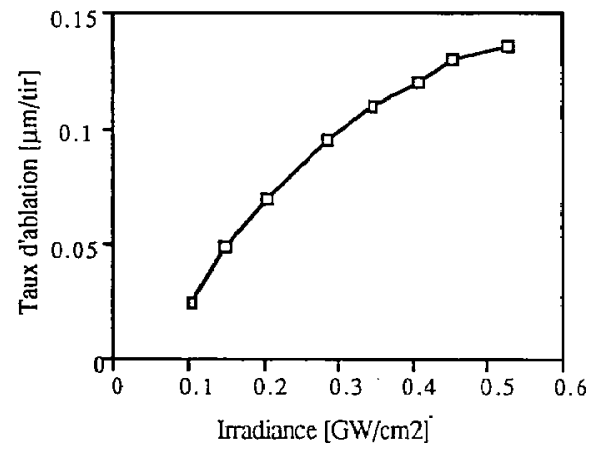

Figure 2. Ablation sur Si3N4 par laser KrF $248 \mathrm{~nm}$ : taux d'ablation fonction de l'irradiance laser.

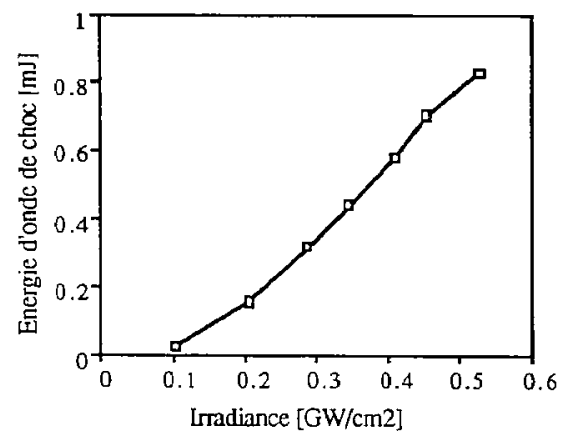

Figure 3. Ablation sur Si3N4 par laser $\mathrm{KrF} 248 \mathrm{~nm}$ : énergie d'onde de choc fonction de l'irradiance laser.

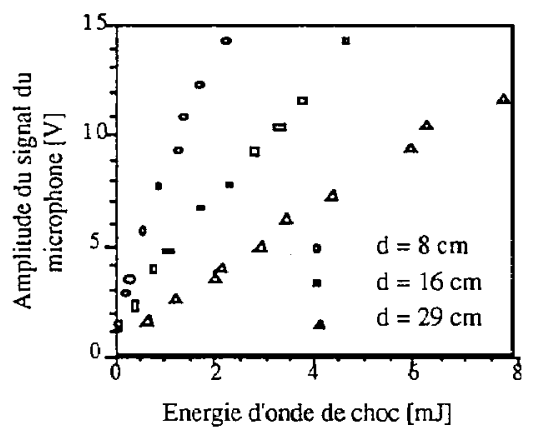

Figure 4. Courbes de calibration du microphone. d est la distance entre microphone et cible.

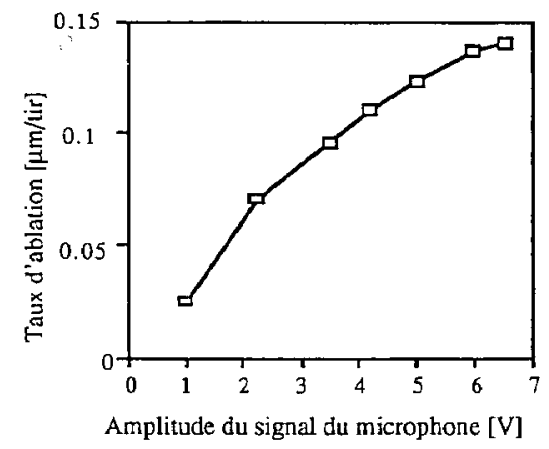

Figure 5. Ablation sur Si3N4 par laser KrF 248nm. Évolution du taux d'ablation en fonction de l'amplitude du signal du microphone; observation à $\mathrm{d}=8 \mathrm{~cm}$

\section{Références}

[1] Laser ablation acoustical monitoring, paper H-V P 40 Symposium E-MRS, Strasbourg Juin 96, to be published in Applied Surface Science.

[2] C. Stauter, "Caractérisation de l'interaction entre un faisceau laser à impulsions courtes et la matière - application au contrôle de procédé d'ablation laser", thèse de doctorat, Université Louis Pasteur, Strasbourg, avril 1996.

[3] D. L. Jones, "Intermediate strength blast wave", Phys. Fluids 11 (8), 1968, p. 1664-1667. 